\title{
Analysis of memory $T$ lymphocyte activity following stimulation with overlapping HLA-A*2402, A*0 I0I and Cw*0402 restricted CMV pp65 peptides
}

\author{
Monica Ghei ${ }^{1}$, David F Stroncek ${ }^{2}$ and Maurizio Provenzano*2,3
}

Address: ${ }^{1}$ Washington University School of Medicine, St. Louis, MO, USA, ${ }^{2}$ Molecular Immunology Sections, Department of Transfusion Medicine, National Institutes of Health, Bethesda, MD, USA and ${ }^{3}$ Institut für chirurgische Forschung und Spitalmanagement, University of Basel, Switzerland

Email: Monica Ghei - gheim@msnotes.wustl.edu; David F Stroncek - dstroncek@dtm.cc.nih.gov; Maurizio Provenzano* - mprovenzano@uhbs.ch

* Corresponding author

Published: 26 May 2005

Journal of Translational Medicine 2005, 3:23 doi:10.1 186/1479-5876-3-23
Received: 02 February 2005

Accepted: 26 May 2005

This article is available from: http://www.translational-medicine.com/content/3/I/23

(C) 2005 Ghei et al; licensee BioMed Central Ltd.

This is an Open Access article distributed under the terms of the Creative Commons Attribution License (http://creativecommons.org/licenses/by/2.0), which permits unrestricted use, distribution, and reproduction in any medium, provided the original work is properly cited.

\begin{abstract}
The continuous efforts aimed at the identification of new immune epitopes across the MHC system has led to the discovery that more than one peptide may be restricted to the same HLA antigen and function as an immune determinant for that association. The aim of this study was to compare the ability of two overlapping peptides, the nonamer (9-mer) cytomegalovirus (CMV) PP65 $341-349$ (QYDPVAALF) and the decamer (I0-mer) CMV pp65 $341-350$ (QYDPVAALFF), and the esadecamer (16-mer) peptide containing both the 9-mer and 10-mer sequences, CMV Pp65 $5_{340-355}$ (RQYDPVAALFFFDIDL), to stimulate and maintain over time a $T$ cell immune reactivation by HLAA*2402, A*0I0I, and CW*0402 cells from CMV-seropositive subjects. The 9-mer, I0-mer, and 16mer peptides effectively stimulated CTLs from HLA-A*2402, HLA-A*0I0I, and HLA-Cw*0402 CMV seropositive donors. This data confirms that both the 9-mer and the 10-mer peptides are promiscuous and are not restricted to a single HLA antigen. CMV Pp65 $341-349$ and CMV Pp65 $341-$ 350 have the ability to produce CMV-specific CTLs in subjects with several different HLA types, presenting a practical advantage over other peptides that are restricted only to a single HLA antigen, and thus being optimal for CMV adoptive immune therapy. Moreover, since the 16-mer peptide encompasses both the 9-mer and 10-mer peptides, it may be better than either of these peptides for CMV adoptive immune therapy.
\end{abstract}

\section{Introduction}

In healthy subjects, primary infection with Cytomegalovirus (CMV) is usually mild or asymptomatic and is effectively controlled by the cell-mediated immune response [1]. However, in immune compromised individuals, such as those with AIDS or after bone marrow transplantation, CMV reactivation is associated with significant morbidity until the individual's immune system is completely recon- stituted [2]. One method of preventing post-transplant CMV infection is adoptive immunotherapy using CMVspecific cytotoxic T cells (CTLs) from the transplant donor [3]. Several HLA class I restricted peptides derived from the immune dominant CMV 65 kd matrix phosphoprotein (pp65) have been shown to produce CMV-specific CTLs. Two overlapping HLA-A*2402 restricted peptides have been described: pp65 $5_{341-349}$ and pp65 $341-350$. These 
peptides are a nonamer (9-mer) and a decamer (10-mer) that overlap except for the last amino acid phenylalanine (F) at the C-terminus [QYDPVAALF(F)]. Despite their similarity, the ability of these peptides to induce a $\mathrm{T}$ cell response has been reported to differ $[4,5]$.

Although it has been generally accepted that a unique CMV peptide is bound and presented by each separate HLA class I molecule, recent studies suggest that certain peptides are more promiscuous and may be presented by more than one HLA class I antigen. In this specific case, the 9-mer pp65 $341-349$ has been shown to stimulate CTLs from both HLA-A*2402 and $\mathrm{Cw}^{*} 0402$ donors [6], while the 10-mer pp65 $341-350$ has been shown to be reactive with both HLA-A*2402 and A*0101 donors [7].

The current investigation sought to compare the potency of these two peptides and determine the optimum peptide size for effective CMV adoptive immune therapy. Both peptides were tested for their ability to stimulate CMVspecific CTLs in HLA-A*2402, HLA-A*0101, and HLA$\mathrm{CW}^{*} 0402$ restriction. In addition, a 16 -mer pp65 $340-355$ that includes both the 9-mer and the 10-mer peptides was tested for its ability to reactivate memory $\mathrm{T}$ cells. This specific 16-mer peptide was selected since it represents the naturally processed peptide that would encompass both the 9-mer and 10-mer peptides. IFN- $\gamma$ mRNA transcript production was measured by in vitro cell culture assays in which the cells were peptide-induced for 3 hours after a 2week in vitro sensitization, while IFN- $\gamma$ protein release was measured using in vitro cell culture supernatant collected at different time points.

The goal of the investigation was to determine whether both the 9-mer and the 10-mer peptides maintain high levels of CTL stimulation over time for all HLA restrictions studied. Moreover, it was important to investigate whether stimulation with the naturally processed 16-mer peptide, followed by re-stimulation by the two smaller peptides embedded within the larger sequence, lead to effective T cell memory immune response.

\section{Materials and methods Peptide selection, synthesis and nomenclature}

Two overlapping peptides derived from the immune dominant CMV $65 \mathrm{kd}$ matrix phosphoprotein (pp65), the nonamer pp65 $341-349$ (QYDPVAALF) and the decamer pp65 $341-350$ (QYDPVAALFF) peptides were used to analyze memory $\mathrm{T}$ lymphocyte activity in PBMCs collected from CMV seropositive donors bearing alleles HLA$A^{*} 2402, A^{*} 0101$, or $\mathrm{CW}^{*} 0402$. The esadecamer pp65 $65_{340-}$ 355 (RQYDPVAALFFFDIDL) sequence that encompasses both pp65 $5_{341-349}$ (QYDPVAALF) and pp65 $5_{341-350}$ (QYDPVAALFF) peptides was selected according to its score, as analyzed by MAPPP (MHC Antigen Peptide Processing
Prediction) [8] based on FRAGPREDICT developed by Holzhütter [9] (Figure 1). The original sequence of 20 amino acids in length (CMV pp 65 $340-359)$, as shown in figure 1 , was reduced to 16 amino acids (CMV pp65 $340-355$ ) in order to allow the complete reconstitution of this sequence. The C-terminus of the peptide was reduced to form a 16-mer sequence and the selection of the 16-mer did not affect the aim of having both 9-mer and 10-mer peptides represented in the sequence. The HLA restriction for this 16 amino acid sequence was not known (Additional File 1).

The three peptides were synthesized by Princeton Biomolecules (Langhorne, PA) with purity from $90 \%$ to $100 \%$ as analyzed by High Performance Liquid Chromatography (HPLC), dissolved at $100 \mu \mathrm{g} / \mathrm{ml}$ in 50\% DMSO and stored at $4{ }^{\circ} \mathrm{C}$. To simplify the peptide nomenclature in this paper we refer to peptide CMV pp65 $5_{341-349}$ (QYDPVAALF) as the 9-mer peptide, to peptide CMV pp65 $341-350$ (QYDPVAALFF) as the 10-mer peptide, and to sequence CMV pp65 $340-355$ (RQYDPVAALFFFDIDL) as the 16-mer peptide.

\section{Donor collection and cell preparation}

Leukocytes were collected from CMV seropositive donors bearing alleles HLA-A*2402, A*0101, or Cw*0402 after obtaining informed consent. The presence of CMV antibodies was analyzed by passive latex agglutination (CMVSCAN kit, Becton Dickinson Microbiology System, Cockeysville, MD). MHC class I genotypes were determined by sequence-specific primer polymerase chain reaction (PCR). Lymphapheresis was performed using a CS3000 Plus blood cell separator (Fenwal Divison, Baxter Health Care, Deerfield, IL), and PBMCs were isolated from the apheresis product by Ficoll (Pharmacia Biotech, Wilkstrom, Sweden) density gradient centrifugation.

\section{PBMCs in vitro sensitization}

The in vitro sensitization involved a 2 -week cell culture in the presence of peptide and IL-2. Briefly, a 2-week in vitro sensitization was performed using PBMCs from CMVseropositive donors. PBMCs from each donor were plated at a concentration of $3 \times 10^{6}$ cells $/ \mathrm{ml}$ in a 24 well $/$ plate with $2 \mathrm{ml}$ RPMI complete medium (10\% AB human serum, penicillin, gentamycin, glutamine, and $1 \%$ HEPES), and directly stimulated with $3 \mu \mathrm{g} / \mathrm{ml}$ of both test and control peptides $\left(1 \mu \mathrm{g} / \mathrm{ml}\right.$ peptides for each $10^{6}$ cells $)$. Recombinant human interleukin-2 (rhIL-2, $100 \mathrm{U} / \mathrm{ml}$, PeproTech, Rocky Hill, NJ) was added every other day to the cell culture. At day 15, each batch of cells was washed and directly re-stimulated in fresh medium with either test or control peptide or not re-stimulated. Three hours after re-stimulation cells were harvested to analyze their IFN- $\gamma$ mRNA transcript production by quantitative real time PCR (qRT-PCR). At three different time points $(24,48$, 


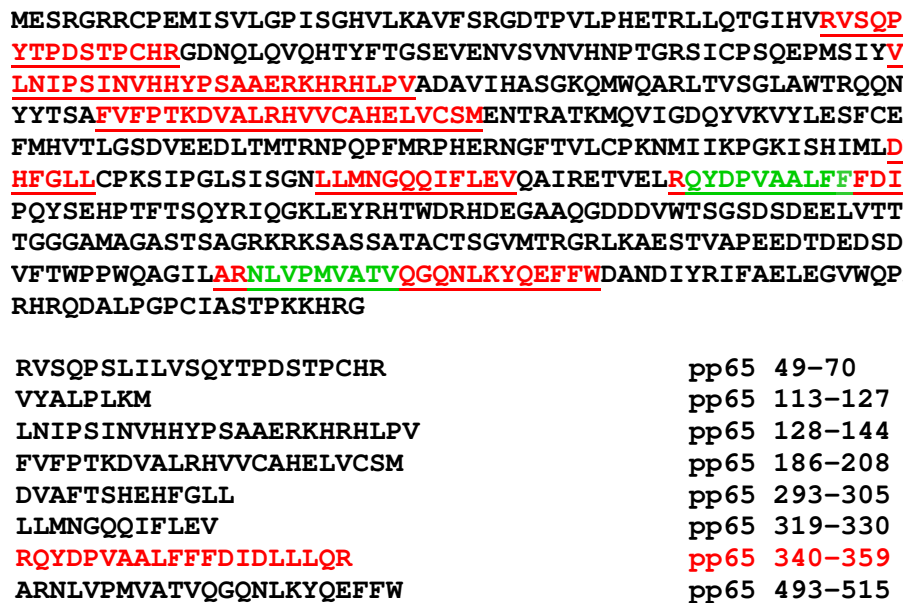

PP65 regions thrs $=0.5 \quad$ MAPPP ( MHC Antigen Peptide Processing Prediction)

\begin{tabular}{l|c|c|c|c|cc} 
SEQUENCE & HRC\% N-term & HRC\% C-term & Peptides & HFC\% mean & \multicolumn{2}{|c}{ peptide cleavage } \\
pp65 49-70 & 0.99 & 0.95 & 10 & 0.62508 & $4>0.6 \max 1$ & $6<0.6 \min 0.5002$ \\
pp65 113-127 & 0.95 & $\mathbf{1 . 0 0}$ & 7 & 0.8512 & $5>0.6 \max 1$ & $2<0.6 \min 0.5003$ \\
pp65 128-144 & $\mathbf{1 . 0 0}$ & 0.95 & 6 & 0.6625 & $2>0.6 \max 1$ & $4<0.6 \min 0.5002$ \\
pp65 186-208 & $\mathbf{1 . 0 0}$ & 0.92 & 10 & 0.77493 & $8>0.6 \max 1$ & $2<0.6 \min 0.5002$ \\
pp65 293-305 & 0.94 & 0.84 & 7 & 0.80037 & $5>0.6 \max 1$ & $2<0.6 \min 0.5004$ \\
pp65 319-330 & 0.96 & 0.91 & 5 & $\mathbf{0 . 8 7 7 9 4}$ & $4>0.6 \max 1$ & $1<0.6 \min 0.5019$ \\
pp65 340-359 & $\mathbf{1 . 0 0}$ & 0.99 & $\mathbf{1 8}$ & 0.7538 & $11>0.6 \max 1$ & $7<0.6 \min 0.5002$ \\
pp65 493-515 & 0.96 & 0.82 & 10 & 0.77032 & $6>0.6 \max 1$ & $4<0.6 \min 0.5000$
\end{tabular}

\section{Figure I}

Scheme of candidate immune regions within the CMV pp65 as analyzed by MAPP. The immune dominant CMV 65 $\mathrm{kd}$ matrix phosphoprotein (pp65) is shown above. The highlighted red sequences within the pp65 represent the most immunogeneic polypeptides, as predicted by the MAPPP algorithm (threshold $\leq 0.5$ ). Their positions in the protein are listed below. The two highlighted green peptides, NLVPMVATV and QYDPVAALF $(F)$ are, respectively, the known HLA-A*020I associated $\mathrm{PP} 5_{495-503}$ and the two HLA-A*2402 associated overlapping peptides Pp65 $341-349$ and $\mathrm{pp} 65_{341-350}$. The table refers to each single polypeptide's degree of cleavage by proteasome activity at either $\mathrm{N}$-terminus or $\mathrm{C}$-terminus. The number of peptides derived from each sequence's proteolitic activity and the corresponding degree of peptide cleavage are also shown. HRC (Highest residue cleavage probability); HFC (Highest fragment cleavage probability).

and 72 hours) after re-stimulation supernatants were collected and tested for IFN- $\gamma$ protein release following the ELISA manufacturer's guidelines.

\section{Quantitative real time PCR (qRT-PCR)}

IFN- $\gamma$ mRNA transcript production by in vitro sensitized PBMCs was evaluated 3 hours after direct peptide reinduction, as previously described [5]. Following 2-week in vitro sensitization (IVS), $2 \times 10^{5}$ PBMCs (final concentration of $1 \times 10^{6}$ cells $/ \mathrm{ml}$ ) were plated in a 96 U-bottom well/plate with $200 \mu \mathrm{l}$ of RPMI complete medium, incubated overnight, and then directly stimulated with $1 \mu \mathrm{g} /$ $\mathrm{ml}$ of specific peptide. After a 3-hour incubation, total RNA was extracted (RNeasy Mini Kit, Qiagen, Valencia, $\mathrm{CA}$ ) and $1 \mu \mathrm{l}$ of synthesized cDNA (Invitrogen, Carlsbad, CA) was used as a template to measure IFN- $\gamma$ mRNA tran- 
scription by qRT-PCR using an ABI Prism 7900 Sequence Detection System (Perkin Elmer, Foster City, CA). Quantitative real time PCR results were reported as the number of IFN- $\gamma$ gene copies normalized by $10^{5} \beta$-actin gene copies. All PCR assays were performed in triplicate and reported as the average. Stimulation index has been applied based on the negative control values.

\section{ELISA}

The release of IFN- $\gamma$ protein by in vitro sensitized PBMCs after peptide re-stimulation was measured using an enzyme-linked immunosorbent assay (ELISA) kit (Endogen, Woburn, MA). Supernatants of peptide-stimulated PBMCs were collected at 24, 48, and 72 hours. ELISA results were extrapolated from a standard curve generated by linear regression. The assays were performed in duplicate and reported as the average.

\section{Results}

IFN- $\gamma$ mRNA transcript production by 9-mer, 10-mer, and I6-mer sensitized PBMCs from HLA-A*2402, A*0 I01, and $C w^{*} 0402$ donors re-stimulated with their cognate determinants

In order to analyze the immediate $\mathrm{T}$ cell immune reactivation to each of the three determinants (9-mer, 10-mer and 16-mer) after 2-week in vitro sensitization, qRT-PCR was employed to quantitate levels of IFN- $\gamma$ mRNA produced by sensitized cells after a 3-hour peptide-induction. Thus, following the 2-week peptide (9-mer, 10-mer, and 16mer) in vitro sensitization, each batch of sensitized cells was re-stimulated with the cognate peptides. Although both 9-mer and 10-mer peptides were able to maintain high levels of stimulation over this time period for all HLA restrictions tested, the 9-mer peptide induced the highest responses in cells expressing HLA-A*2402 (S.I. 4.07-528) or HLA-Cw*0402 (S.I. 4.15-483) while the 10-mer peptide induced the highest responses in cells expressing HLA-A*2402 (S.I. 3.5-528) or HLA-A*0101 (S.I. 8.25$615)$. The 16-mer peptide was also able to stimulate $T$ cells from all HLA-A*2402, A*0101 and Cw*0402 donors (S.I. $6.95,4.96,5.02)$ at levels that were approximately equal to the average of those induced by each single 9-mer and 10-mer induction (Figure 2A).

\section{IFN- $\gamma$ mRNA transcript production by I6-mer peptide- sensitized PBMCs from HLA-A*2402, A*0 I0I, and $C W^{*} 0402$ donors re-stimulated with either the 9-mer or the I0-mer determinant}

In order to analyze the immediate reactivation of memory T lymphocytes following the 16-mer peptide sensitization, the 2-week in vitro 16-mer peptide sensitized cells were re-stimulated with either 9-mer or 10-mer peptide. Thus, qRT-PCR was employed to quantitate levels of IFN$\gamma$ mRNA produced by sensitized cells after a 3-hour peptide-induction. Compared to the previous results, as in figure $2 \mathrm{~A}$, that demonstrated the 9-mer peptide's specificity for HLA-A*2402 and HLA-Cw*0402, the re-stimulation of 2-week in vitro 16-mer peptide sensitized cells with the 9-mer peptide confirmed the 9-mer peptide's specificity for HLA-A*2402 and HLA-Cw*0402 plus its ability to enhance the $\mathrm{T}$ lymphocytes reactivity in donors bearing HLA-A*0101 (S.I. 3.96-507). Similarly, the re-stimulation of 2-week in vitro 16-mer peptide sensitized cells with the 10-mer peptide confirmed this peptide's specificity for HLA-A*0101 while specifically enhancing the T cell reactivity in HLA-A*2402 donors (S.I. 6.12-236). The HLA$\mathrm{CW}^{*} 0402$ specificity was also confirmed and maintained for the 10-mer peptides (3.58-467), probably adding a new HLA association to this CMV peptide (Figure $2 \mathrm{~B}$ ).

\section{IFN- $\gamma$ protein release by 9-mer, 10-mer, and I6-mer peptide-sensitized PBMCs from HLA-A*2402, A*0 I 01 , and $C w^{*} 0402$ donors re-stimulated with their cognate determinants}

Following 2-week in vitro sensitization with 9-mer, 10 mer or 16-mer peptides of PBMCs from HLA-A*2402, $\mathrm{A}^{*} 0101$, and $\mathrm{CW}^{*} 0402$ donors, supernatants were collected from cell cultures at 24, 48 and 72 hours after the re-stimulation of each sensitized batch of cells with the cognate peptides. Despite the fact that both the 9-mer and 10-mer peptides were able to reactivate cells from each of the three donors at time 0 (Figure 2A), the 9-mer peptide was weaker than the 10-mer peptide in maintaining a consistent immune $\mathrm{T}$ response over time in both the HLA$A^{*} 2402$ and HLA-A*0101 donors. In contrast, the 9-mer peptide was stronger than the 10-mer in stimulating and maintaining a $\mathrm{T}$ immune reactivation and response over time in donors bearing HLA-Cw*0402. The stimulation with the 16-mer peptide induced and maintained consistent immune $\mathrm{T}$ cell reactivation in all donors tested, as assessed by levels of IFN- $\gamma$ protein production when compared to the positive control (Figure 3).

\section{IFN- $\gamma$ protein release by I6-mer sensitized PBMCs from} HLA-A*2402, A*0IOI, and CW*0402 donors re-stimulated with either 9-mer or 10-mer determinant

Following 2-week in vitro sensitization of PBMCs from HLA-A*2402, A*0101, and $\mathrm{Cw}^{*} 0402$ donors with the 16mer sequence, supernatants were collected from cell culture at 24, 48 and 72 hours after re-stimulation of 16-mer peptide-sensitized cells with either 9-mer or 10-mer peptides. It seems that in all donors the re-stimulation of 16mer in vitro sensitized cells with the 9-mer and the 10-mer peptides was able to better induce IFN- $\gamma$ protein production than 9-mer or 10 -mer in vitro sensitized cells that were stimulated with the cognate peptide either by enhancing cytokine protein production at each time point or by better maintaining cytokine protein release over time. Specifically, this was seen in both HLA-A*2402 and HLA-A*0101 donors following 9-mer peptide induction 
A

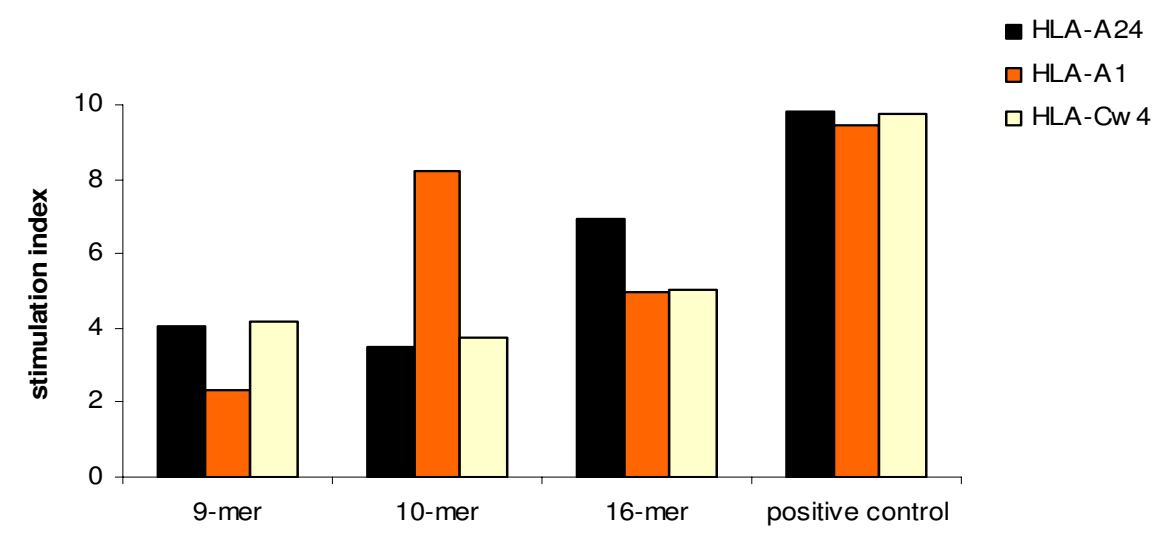

B

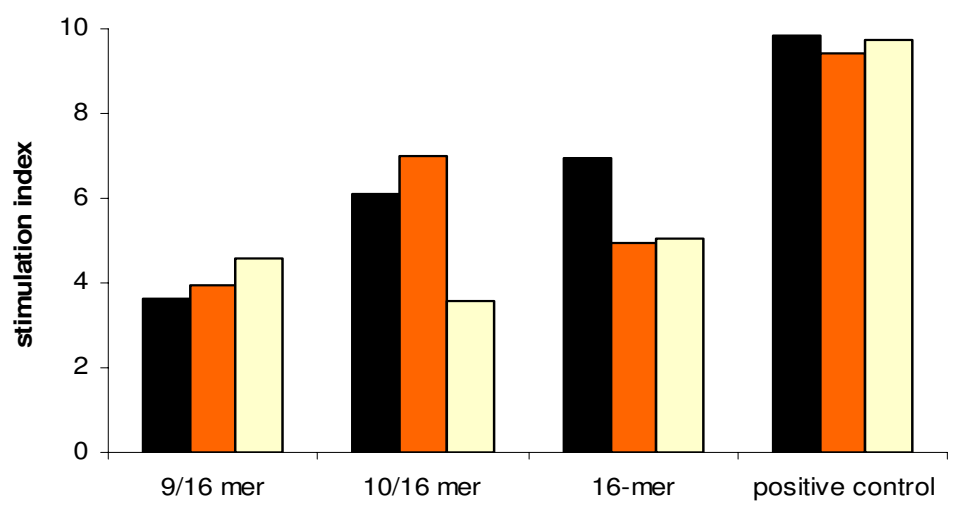

Figure 2

IFN- $\gamma$ mRNA transcript production by qRT-PCR. The ability of the three CMV peptides; Pp $65_{34 I-349}$, Pp $65_{34 I-350}$, and $\mathrm{PP}_{340-355}$ to reactivate an immune $\mathrm{T}$ lymphocyte response after their cognate re-stimulation of self-induced PBMCs (A) or after restimulation with each peptide of 16-mer induced PBMCs (B) was analyzed by qRT-PCR. Levels of IFN- $\gamma$ mRNA transcript were quantitated after a 3-hour peptide induction. The results were performed in triplicate and reported as the average. Levels of IFN- $\gamma$ mRNA transcript were normalized by levels of $\beta$-actin mRNA transcript and the final values were indicated as the ratio over the negative control.

and in the HLA-Cw*0402 donor following 10-mer peptide induction (Figure 4 ).

\section{Discussion}

The adoptive transfer of immunodominant $\mathrm{T}$ lymphocytes to CMV-infected transplanted patients represents one of the treatments of choice to clear the CMV disease and to quickly reconstitute the lost balance between the CMV infection and the immune system [1012]. The use of specific immune peptides to detect and expand immunocompetent $\mathrm{T}$ cells is an important tool that has been already applied in several clinical trials $[13,14]$. Once CMV epitope mapping had been initiated and led to the identification of epitopes encompassing several HLA class I antigens, great effots were devoted to the identification of HLA cross-reactivity of known immune determinants and the identification of new determinants. In this specific case, the two overlapping peptides pp65 $341-349$ (QYDPVAALF) and pp65 $341-350$ (QYDPVAALFF) have been shown to have a marked cross- 


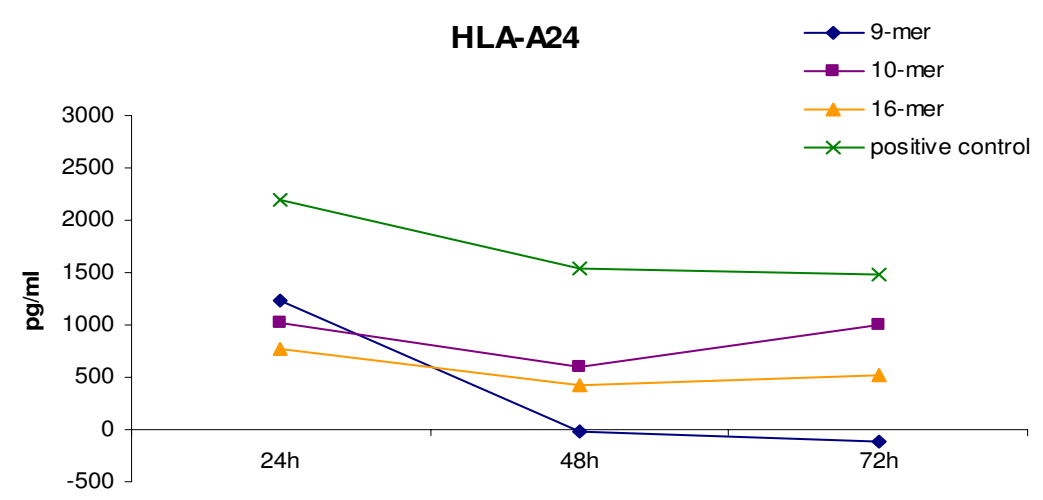

HLA-A1

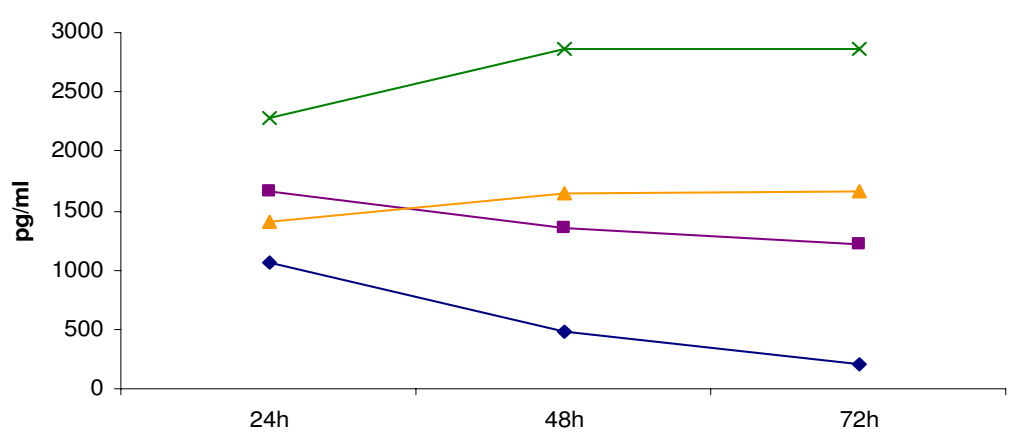

HLA-Cw4

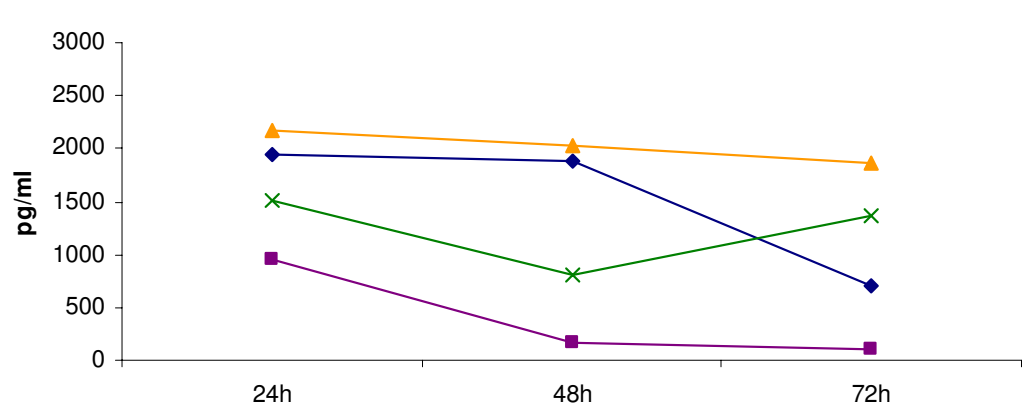

Figure 3

IFN- $\gamma$ protein release by ELISA following re-stimulation of peptide (9-mer, I0-mer, and I6-mer) sensitized PBMCs from all donors with their cognate determinants. Supernatants of peptide (9-mer pp65 $341-349$, 10-mer $\mathrm{pp} 65_{341-}$ 350 , and I6-mer $\mathrm{Pp} 65_{340-355}$ ) sensitized PBMCs from CMV seropositive donors HLA-A*2402, A*010I, and Cw*0402 were collected at three time point of 24,48 , and 72 hours after cell re-stimulation with each cognate peptide: 9-mer (blue segment), I0mer (purple segment), and 16-mer (orange segment). The figure shows the peptide induction and the maintenance over time of $T$ lymphocyte reactivation after cognate peptide recall compared to the positive control (green segment) for each individual HLA association: HLA-A24 (top), HLA-AI (middle), HLA-Cw4 (bottom). 
HLA-A24

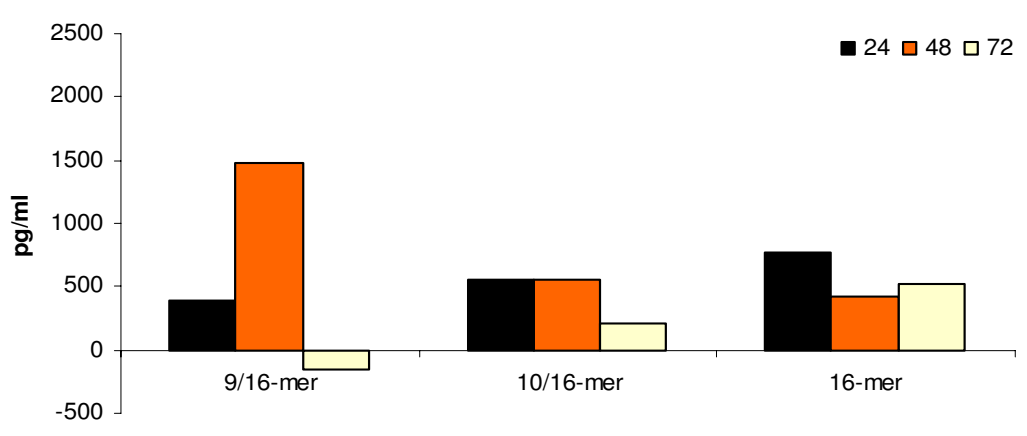

HLA-A1

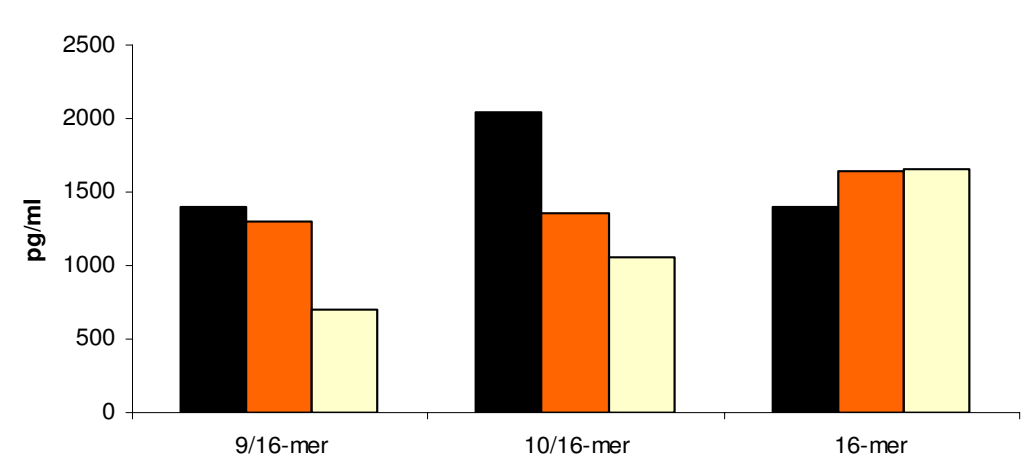

HLA-Cw4

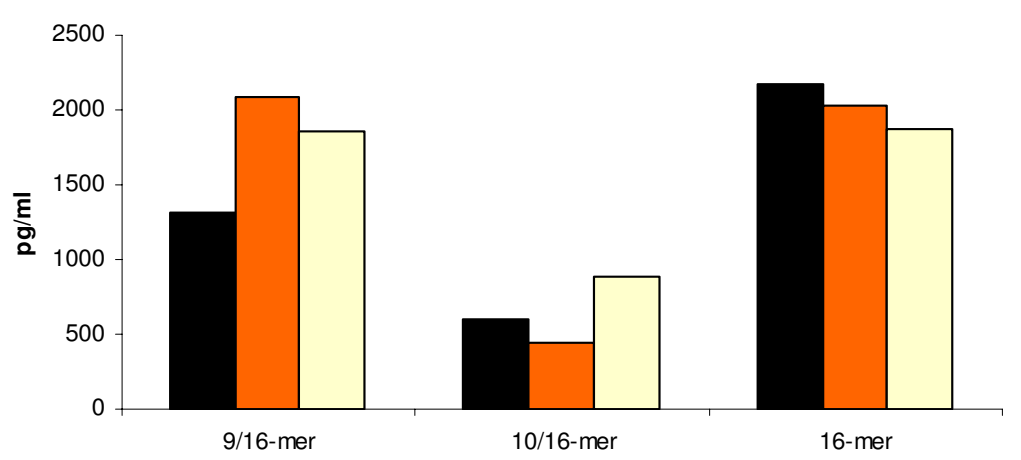

Figure 4

IFN- $\gamma$ protein release ELISA following re-stimulated of I 6-mer sensitized PBMCs from all donors with either the 9-mer or I 0-mer determinant. Supernatants of 16-mer Pp65 $340-355^{-}$-sensitized PBMCs from CMV seropositive donors HLA-A*2402, $A^{*} 0101$, and $C w^{*} 0402$ were collected at the three time points of 24 hours (black), 48 hours (orange), and 72 hours (yellow) after cell re-stimulation with either the 9-mer $\mathrm{Pp} 65_{341-349}$ or 10-mer Pp65 34 I-350 Peptide. The figure shows the peptide induction and maintenance over time of the T lymphocyte reactivation from 16-mer Pp65 $340-355^{-}$-sensitized PBMCs after either 9-mer Pp65 $341-349$ (9/16) or 10-mer Pp65 34I-350 (10/16) peptide recall compared to the specific 16-mer Pp65 $340-355$ re-induction ( 16 ) for each individual HLA association: HLA-A24 (top), HLA-AI (middle), HLA-Cw4 (bottom). 
reactivity. First, it was shown that pp65 $65_{341-349}$ is restricted to HLA-A*2402 and Cw*0402 and pp65 $5_{341-350}$ to HLA$A^{*} 2402$ and $A^{*} 0101[6,7]$. In this study a new HLA specificity for the 9-mer to HLA-A*0101 and for the 10-mer to HLA-Cw*0402 is well described. Furthermore, this study showed that both peptides were able to effectively stimulate immune $\mathrm{T}$ cell responses from HLA-A*2402, A*0101 and $\mathrm{CW}^{*} 0402$ donors, confirming that these peptides are promiscuous and not restricted to a single HLA type. While both the 9-mer and 10-mer peptides are able to maintain high levels of stimulation over time in HLAA*2402 donors, both are also able to induce and maintain an immune reactivation in donors bearing their other noted HLA associations. In fact, the 9-mer is better able to maintain stimulation in $\mathrm{HLA}-\mathrm{Cw}^{*} 0402$ donors and the 10 -mer is better able to do so in HLA-A*0101 donors.

Interestingly, another new finding emerged: both the 9mer and 10-mer peptides were able to induce a population of restricted $\mathrm{T}$ lymphocytes from a cell population that had been in vitro sensitized with a peptide sequence that encompassed the two peptides. The 16-mer peptide is able to effectively stimulate T cells from HLA-A*2402, $\mathrm{A}^{*} 0101$ and $\mathrm{CW}^{*} 0402$ at levels that are well maintained over time. In particular, it seems that the 9-mer and 10mer peptide reactivation of cells previously sensitized with the 16-mer peptide results in enhanced T cell reactivation in all donors. Although these results need to be confirmed and validated by further in vivo studies, we speculate that the use of this 16-mer region rather than the single 9-mer or 10-mer peptides would be advantageous in clinical modalities such as adoptive transfer of epitopespecific T lymphocytes or epitope-specific vaccinations. These results support the potential use of the 16-mer peptide in CMV adoptive immune therapy [15].

\section{Additional material}

\section{Additional File 1}

Table 1. to go DOC

Click here for file

[http://www.biomedcentral.com/content/supplementary/1479-

5876-3-23-S1.doc]

\section{Acknowledgements}

This research was conducted through the generous support of the NIH

Summer Research Fellowship Program for medical/dental students.

\section{References}

I. Zaja JA: Cytomegalovirus infection. In Hematopoietic Cell Transplantation Edited by: Thomas ED, Blume KG, Forman SJ. Blackwell Science; 1999:560-583.

2. Bronke C, Palmer NM, Jansen CA, Westerlaken GH, Polstra AM, Reiss P, Bakker M, Miedema F, Tesselaar K, Baarle D: Dynamics of Cytomegalovirus (CMV)-Specific T Cells in HIV-I-Infected
Individuals Progressing to AIDS with CMV End-Organ Disease. J Infect Dis 2005, I 9 I:873-880.

3. Rauser G, Einsele H, Sinzger C, Wernet D, Kuntz G, Assenmacher M, Campbell JD, Topp MS: Rapid generation of combined CMVspecific CD4+ and CD8+ T-cell lines for adoptive transfer into recipients of allogeneic stem cell transplants. Blood 2004, 103:3565-3572.

4. Kuzushima K, Hayashi N, Kimura H, Tsurumi T: Efficient identification of HLA-A*2402-restricted cytomegalovirus-specific CD8(+) T-cell epitopes by a computer algorithm and an enzyme-linked immunospot assay. Blood 200I, 98:|872-|88I.

5. Provenzano M, Mocellin S, Bettinotti M, Preuss J, Monsurro V, Marincola FM, Stroncek D: Identification of immune dominant cytomegalovirus epitopes using quantitative real-time polymerase chain reactions to measure interferon-gamma production by peptide-stimulated peripheral blood mononuclear cells. J Immunother 2002, 25:342-35I.

6. Kondo E, Akatsuka $Y$, Kuzushima K, Tsujimura K, Asakura S, Tajima $\mathrm{K}$, Kagami Y, Kodera Y, Tanimoto M, Morishima Y, Takahashi T: Identification of novel CTL epitopes of CMV-pp65 presented by a variety of HLA alleles. Blood 2004, 1 03:630-638.

7. Provenzano M, Lim JB, Mocellin S, Monsurro V, Bettinotti M, Marincola FM, Stroncek DF: The matrix protein pp65(34I-350): a peptide that induces ex vivo stimulation and in vitro expansion of CMV-specific CD8+ T cells in subjects bearing either HLA-A*2402 or A*0 I0I allele. Transfusion 2003, 43:I567-I57.

8. Hakenberg J, Nussbaum AK, Schild H, Rammensee HG, Kuttler C, Holzhutter HG, Kloetzel PM, Kaufmann SH, Mollenkopf HJ: MAPPP: MHC class I antigenic peptide processing prediction. Appl Bioinformatics 2003, 3:155- I58.

9. Holzhütter HG, Kloetzel PM: A kinetic model of vertebrate 205 proteasome accounting for the generation of major proteolytic fragments from oligomeric peptide substrates. Biophysical J 2000, 79: I | 96- 1205.

10. Riddell S, Greenberg P: Principles for adoptive T cell therapy of human viral disease. Annu Rev Immunol I995, I 3:545-586.

II. Einsele H, Roosnek E, Rufer N, Sinzger C, Riegler S, Loffler J, Grigoleit U, Moris A, Rammensee HG, Kanz L, Kleihauer A, Frank F, Jahn G, Hebart H: Infusion of cytomegalovirus (CMV)-specific T cells for the treatment of CMV infection not responding to antiviral chemotherapy. Blood 2002, 99:3916-3922.

12. Lim JB, Kwon OH, Kim HS, Kim HO, Choi JR, Provenzano M, Stroncek D: Adoptive immunotherapy for cytomegalovirus (CMV) disease in immunocompromised patients. Yonsei Med J 2004, 45: $18-22$.

13. Szmania S, Galloway A, Bruorton M, Musk P, Aubert G, Arthur A, Pyle H, Hensel N, Ta N, Lamb L Jr, Dodi T, Madrigal A, Barrett J, HensleeDowney J, van Rhee F: Isolation and expansion of cytomegalovirus-specific cytotoxic T lymphocytes to clinical scale from a single blood draw using dendritic cells and HLA-tetramers. Blood 200I, 98:505-5I2.

14. Watanabe N, Kamachi Y, Koyama N, Hama A, Liang J, Nakamura Y, Yamamoto T, Isomura M, Kudo K, Kuzushima K, Kojima S: Expansion of human CMV-specific cytotoxic T lymphocytes to a clinical scale a simple culture system using tetrameric HLApeptide complexes. Cytotherapy 2004, 6:5 I 4-522.

15. Trivedi D, Williams RY, O'reilly RJ, Koehne G: Generation of Cytomegalovirus (CMV)-specific T lymphocytes using protein-spanning pools of pp65-derived pentadecapeptides for adoptive immunotherapy. Blood 2005, 105:2793-2801. 